\title{
Vegetation Analysis of Kanewal Wetland, Gujarat
}

\author{
Juhi Gajjar*1, Dr. Hitesh Solanki²
}

${ }^{* 1}$ Ph.D. Research scholar, Department of Botany, Gujarat University, Ahmedabad, Gujarat, India ${ }^{2}$ Professor, Department of Botany, Gujarat University, Ahmedabad, Gujarat, India

\begin{abstract}
Article Info

Volume 8, Issue 3

Page Number : 259-266

Publication Issue

May-June-2021

Article History

Accepted : 14 May 2021

Published : 22 May 2021

Wetland provides various plant species. These plants are use as food, fodder, timber, it is use as medicine, provide shelter for birds, as well as water-birds, migratory birds, local animals and human. Kanewal Wetland is an Irrigation Reservoir. Vegetation of Kanewal is very rich in diversity. Study shows presence of Different species of Angiosperms and pteridophytes. Vegetation analysis were done for year 2018-2019 for every season. Families of species are classified in to monocot and dicot. Many plant species grown there which is very important medicinally as well.
\end{abstract}

Keywords : Wetland, Vegetation, Species, Food

\section{INTRODUCTION}

Wetlands are the most productive ecosystem of the earth (Ghermandi et al., 2008). Wetland ecosystem forms an important environment for aquatic, semiaquatic and moisture loving floral and faunal associations. They act as bio filter, as they intake large amount of organic as well as inorganic nutrients from the eutrophic water bodies nutrients enriched pollutant through various dynamic processes, e.g. Water cycle, nutrient cycle and food chain, therefore, kwon as "kidney of landscape" or "biological super market" by the experts or the areas where the soil is saturated with water are crucial incubators known for high diversity (Allen-Diaz et al., 2004). Wetland provides various plant species. These plants are use as food, fodder, timber, it is as medicine, provide shelter for birds. It is important to know value of that plants. So that we can understand the value of that plant by vegetation analysis. Nowadays wetlands destroy by various activities: urbanization, land use change, drainage to agriculture use, and pollution from industrial effluents and agricultural runoff, climate change and viability. Loss in wetland area results in adverse impact on the key functions (ecosystem goods and services) performed by wetlands (Zedler and Kercher, 2005). Kanewal Wetland is an Irrigation Reservoir. This is man-made wetland having freshwater that is retained almost throughout the year as besides rainfall, waters from canal networks like those of Mahi and Narmada are also supplied to it. Its water used for irrigation and drinking purposes. There is Vast sheet of open water fragmented by yellow-brown blanket of live or decomposing submerged vegetation; Lush green, dense and extensive underwater meadows of submerged hydrophytes, three large islands with one having farmlands and one having date-palm trees with 
heronry, pink-green carpet of lotus and dense growth of typha angustata, and there are various migratory water birds surrounding area of Kanewal wetland.

\section{METHODOLOGY}

\subsection{Study area: -}

Kanewal (22028'00" N and 72032'00" E) is located in Tarapur taluka, Anand district the distance of about $60 \mathrm{~km}$ from Anand, $80 \mathrm{~km}$ from Ahmedabad. It is situated $30 \mathrm{~km}$ north of the tip of Gulf of Khambhat and around $50 \mathrm{~km}$ south east of the southern portion of Nalsarovar the only Ramsar site in the state.

\subsection{Map of Study area:-}

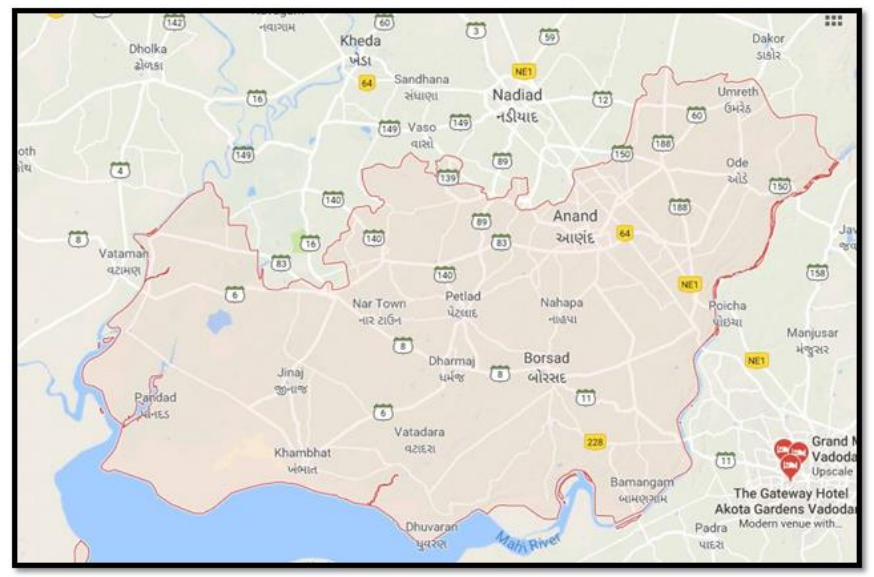

Image: 1: Map of Anand district, Gujarat

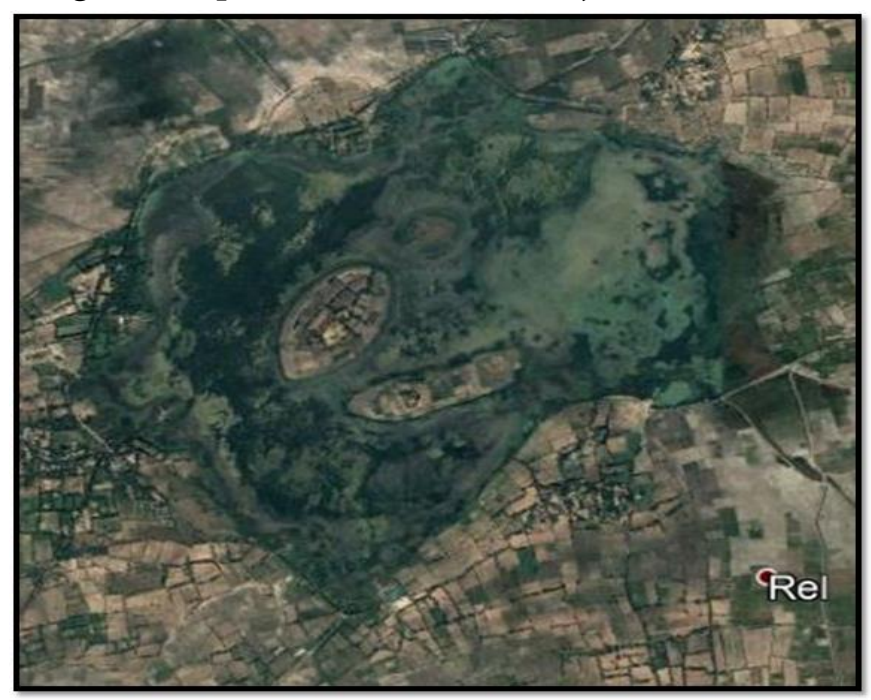

Image: 2: Map of Kanewal Lake

For present study Kanewal wetland was visited in 2018-2019 during every seasons. It takes 1 week during every seasons. Plants were recorded by field survey. Random quadrate laid for analysis of vegetation. Vegetation were divided in to three layers: Upper layer, middle layer and ground layer. Aquatic plants were divided in to four parts: Under water plants, rooted plants with floating leaves, free floating leaves and emergent plants.

\section{RESULT AND DISCUSSION}

\subsection{Vegetation layer of Kanewal wetland}

Vegetation can be divided in to three layers, upper tree layer, middle shrub and climber layer, lower herbaceous layer.

\section{- Upper layer:}

Acacia farnesiana (L.) Willd. Acacia leucophloea (roxb.) willd., Acacia nilotica (L.) Delile subsp. adstringens (Schum. \& Thonn.) Roberty, Aerva lanata (L.) Juss. Delonix elata (L.) Gamble, Eucalyptus globulus Labill. Ficus racemosa L., Ficus religiosa L., Gliricidia sepium (Jacq.) Walp. Holoptelea integrifolia Planch, Melia azedarach L., Pongamia pinnata (L.) Pierre, Prosopis julifera (Sw.) DC.,Senna siamea (Lam.) H. S. Irwin \& Barneby are the tree species, out these Eucalyptus globulus, and Melia azedarach, Senna Siamea are the cultivated species at Kanewal.

\section{- Middle layer:}

Anisomeles indica (L.) O. ktze., Calotropis gigantea (L) Dryand, Calotropis procera (Aiton) Dryand, Jatropha gossypifolia L., Lantana camara L. var. aculeata (L.) Mold, , Ocimum gratissimum L, Opuntia elatior Mill, Peristrophe paniculata (Forssk) Brummitt, Phyllanthus reticulatus Poir, Plumbago auriculata Lam. , Salvadora persica L., Senna occidentalis (L.) Link, Solanum indicum L, Solanum nigrum L, Solanum diphyllum L., Ziziphus rugosa Lam. Out of these lantana camara is invasive species. Cissus trifoliata (L.) L., Ctenolepis garcini (L.) C. B. Clarke, Dregea volubilis (L f) Benth ex Hk f,. Luffa acutangula (L) 
Roxb, Operculina turpethum (L.) Silva Manso, Oxystelma esculentum (L. f.) Sm., Pergularia daemia (Forssk) Chiov, Rivea hypocrateriformis Choisy, Teramnus labialis (L f) Spreng, Teramnus mollis Benth. that are the climber species found in the study area. Operculina turpethum and cissus trifoliata aggressive species found at Kanewal wetland.

\section{- Ground layer:}

Alternanthera sessilis (L.) R. Br. ex DC., Alysicarpus vaginalis (L.) DC., Bergia odorata Edgew., Boerhavia diffusa L, Cynodon dactylon (L) Pers. Desmodium triflorum (L.) DC. Eleusine indica (F) Gaerth, Indigofera tinctoria L. are the most common species. Apluda mutica L., Aristida hystrix L. f, Digitaria ciliaris (Retz.) Koeler, Eragrostis uniloides (Retz. ) Nees ex Steud., Panicum spp are major grass species at Kanewal Wetland.

\subsection{Aquatic plants in Kanewal wetland}

Nelumbo nucifera, Potamogenton pectinatus, Potamogeton perfoliax, Potamogeton nodosus, Nympodes indica, Nymphea pubescence, Utricularia stellaris, Hydrilla verticilata, Najas marina, Najas minor, Ottelia spp are major aquatic angiosperms.

1) Underwater Plants: Vallisnera spiralis, Hydrilla verticellata, Najas spp., Char asp. (macro-alga), Nitella sp. (a macro-alga), Ottelia alismoides and etc.;

2) Rooted Plants with Floating Leaves: Nymphoides cristatum, M. indicum, Nelumbo nucifera, Nymbphaea sp., Ipomoea aquatic;

3) Free Floating Plants: Eichhornia crassipes. Lemna spp., Wolffia globose, Azolla pinnata;

4) Emergent Plants: Typha aungustata, Polygonum sp., Limnophyton obtusifoliuym, Sedges

\subsection{Vegetation of Kanewal Wetland:-}

Table: 1: List of plants from Kanewal wetland

\begin{tabular}{|l|l|l|l|l|}
\hline Sr. No & Botanical name & Local name & Family & Habit \\
\hline 1 & Acalypha indica L. & $\begin{array}{l}\text { Vaichikato, Dadari, } \\
\text { Dadarjo, Dadaro }\end{array}$ & Euphorbiaceae & Herb \\
\hline 2 & $\begin{array}{l}\text { Launaea procumbens (Roxb.) } \\
\text { Ramayya \& Rajagopal }\end{array}$ & Moti Bhonpatri & Asteraceae & Herb \\
\hline 3 & Acacia farnesiana (L.) Willd. & Needle brush & Mimosaceae & Tree \\
\hline 4 & Acacia leucophloea (roxb.) willd. & Dev Baval & Mimosaceae & Tree \\
\hline 5 & $\begin{array}{l}\text { Acacia nilotica (L.) Delile subsp. } \\
\text { adstringens (Schum. \& Thonn.) } \\
\text { Roberty }\end{array}$ & Babul & Mimosaceae & Tree \\
\hline 6 & Acanthospermum hispidum DC. & Goat's Head Plant & Asteraceae & Herb \\
\hline 7 & Achyranthes aspera L. var. aspera & Aghedo, Aapamarg & Amaranthaceae & Herb \\
\hline 8 & Achyranthus aspera L. & Anghedi, Anghedo & Amaranthaceae & Herb \\
\hline 9 & Aerva lanata (L.) Juss. & Kapuri, Gorkh-ganjo & Amaranthaceae & Tree \\
\hline 10 & $\begin{array}{l}\text { Alternanthera polygonodius (L) } \\
\text { R Br ex Sweet }\end{array}$ & Jal Jambvo, & Amaranthaceae & Herb \\
\hline 11 & $\begin{array}{l}\text { Alternanthera sessilis (L.) R. Br. } \\
\text { ex DC. }\end{array}$ & Pani ni Bhaji & Amaranthaceae & Herb \\
\hline
\end{tabular}




\begin{tabular}{|c|c|c|c|c|}
\hline 12 & $\begin{array}{l}\text { Alternanthera sessilis (L.) R.Br. } \\
\text { ex DC }\end{array}$ & & Amarathaceae & Herb \\
\hline 13 & Alysicarpus vaginalis (L.) DC. & $\begin{array}{l}\text { Gadar Samervo, Bhoy } \\
\text { samervo }\end{array}$ & Fabaceae & Herb \\
\hline 14 & Amaranthus spinosus L & Kantemath & Amaranthaceae & Herb \\
\hline 15 & Amaranthus spinosus L & Kantado-Dhimdo & Amaranthaceae & Herb \\
\hline 16 & $\begin{array}{l}\text { Andrographis } \quad \text { paniculata } \\
\text { (Burm.f.) Nees }\end{array}$ & Ruchhado -Samervo & Acanthaceae & Herb \\
\hline 17 & Anisomeles indica (L.) O. ktze. & Chodharo & Lamiaceae & Shrub \\
\hline 18 & Apluda mutica L. & Ponai & Poaceae & Grass \\
\hline 19 & Aristida hystricula L. f & Lapdo & Poaceae & Grass \\
\hline 20 & Bacopa monnieri (L) Wettst & Bam & Scrophulariaceae & Herb \\
\hline 21 & Bergia odorata Edgew. & Lavaru, Ropatri & Elatinaceae & Herb \\
\hline 22 & Blepharis boerhaviifolia Pers. & Chopa ni vel & Acanthaceae & Herb \\
\hline 23 & Boerhavia diffusa $\mathrm{L}$ & Satodi & Nyctaginaceae & Herb \\
\hline 24 & Calotropis gigantea (L) Dryand & Aakdo & Asclepiadaceae & Shrub \\
\hline 25 & $\begin{array}{l}\text { Calotropis procera } \quad \text { (Aiton) } \\
\text { Dryand }\end{array}$ & Nano akado & Asclepiadaceae & Shrub \\
\hline 26 & Cissus trifoliata (L.) L. & Marine ivy & Vitaceae & $\begin{array}{l}\text { Climbe } \\
\mathrm{r}\end{array}$ \\
\hline 27 & Cleome viscosa L. & Pili-tilvan & Capparaceae & Herb \\
\hline 28 & Commelina benghalensis L. & Shishmuliu & Commelinaceae & Herb \\
\hline 29 & Commelina diffusa Burm $\mathrm{f}$ & $\begin{array}{ll}\text { Kanshura, } & \text { Creeping } \\
\text { Dayflower } & \end{array}$ & Commelinaceae & Herb \\
\hline 30 & Corchorus aestuans L & Chunch & Tiliaceae & Herb \\
\hline 31 & Corchorus capsularis L & Chunchi & Tiliaceae & Herb \\
\hline 32 & Corchorus fascicularis Lam & Ubhi-baufali & Tiliaceae & Herb \\
\hline 33 & Corchorus olitorius L & Jute, Chhunchhdo & Tiliaceae & Herb \\
\hline 34 & Crotalaria annua Milne-Redh. & & Fabaceae & Herb \\
\hline 35 & Crotolaria medicaginea Lam & Ranmethi & Fabaceae & Herb \\
\hline 36 & $\begin{array}{lllll}\text { Ctenolepis garcini } & \text { (L.) } & \text { C. } & \text { B. } \\
\text { Clarke } & & & & \\
\end{array}$ & Garcen's Bur Cucumber & Cucurbitaceae & $\begin{array}{l}\text { Climbe } \\
\mathrm{r}\end{array}$ \\
\hline 37 & $\begin{array}{l}\text { Cucumis melo L. subsp. agrestis } \\
\text { (Naudin) Pangalo var. agrestis } \\
\text { Naudin }\end{array}$ & Muskmelon & Cycurbitaceae & $\begin{array}{l}\text { Climbe } \\
\mathrm{r}\end{array}$ \\
\hline 38 & Cynodon dactylon (L) Pers & Darbh & Poaceae & Herb \\
\hline 39 & Cyperus bulbosus Vahl & & Cyperaceae & Sedge \\
\hline 40 & Cyperus compressus L & Poorland -flatsedge & Cyperaceae & Sedge \\
\hline 41 & Cyperus difformis L. & $\begin{array}{l}\text { Small flower umbrella } \\
\text { sedge, Rice sedge }\end{array}$ & Cyperaceae & Sedge \\
\hline 42 & Cyperus rotundus L. & Chido, Moth, Chiyo & Cyperaceae & Sedge \\
\hline
\end{tabular}


Juhi Gajjar et al Int J Sci Res Sci \& Technol. May-June-2021, 8 (3) : 259-266

\begin{tabular}{|c|c|c|c|c|}
\hline 43 & $\begin{array}{l}\text { Dactyloctenium aegyptium (L) P } \\
\text { Beauv }\end{array}$ & Makra, Crow foot grass & Poaceae & Herb \\
\hline 44 & Delonix elata (L.) Gamble & $\begin{array}{l}\text { Creamy Peacock } \\
\text { Flower, Safed gulmohar }\end{array}$ & Fabaceae & Tree \\
\hline 45 & Desmodium triflorum (L.) DC. & Ran methi & Fabaceae & Herb \\
\hline 46 & Digitaria ciliaris (Retz.) Koeler & Bamboo grass & Poaceae & Grass \\
\hline 47 & $\begin{array}{l}\text { Dregea volubilis (L f) Benth ex } \\
\text { Hk f }\end{array}$ & Dodi, Moti Dodi & Asclepiadaceae & $\begin{array}{l}\text { Climbe } \\
\mathrm{r}\end{array}$ \\
\hline 48 & Echinops echinatus Roxb. & Utkanto, Shuliyo & Asteraceae & Herb \\
\hline 49 & Eleusine indica $(\mathrm{F})$ Gaerth & Nagli & Poaceae & Herb \\
\hline 50 & Eragrostis tenella L & Kalavo & Poaceae & Herb \\
\hline 51 & $\begin{array}{l}\text { Eragrostis uniloides (Retz) Nees } \\
\text { ex Steud. }\end{array}$ & Chinese Lovegrasss & Poaceae & Grass \\
\hline 52 & Eucalyptus globules Label. & Nilgiri & Myrtaceae & Tree \\
\hline 53 & Euphorbia hirta L & Asthmaweed & Euphorbiaceae & Herb \\
\hline 54 & Euphorbia hypericifolia L. & Dhudheli & Euphorbiaceae & Herb \\
\hline 55 & Ficus racemosa L. & Umbar, Audumbar & Moraceae & Tree \\
\hline 56 & Ficus religiosa $\mathrm{L}$. & $\begin{array}{l}\text { Peepal, Pimpal, Ashta, } \\
\text { Ashit }\end{array}$ & Moraceae & Tree \\
\hline 57 & Gliricidia sepium (Jacq.) Walp. & Maxican lilac & Fabaceae & Tree \\
\hline 58 & Holoptelea integrifolia Planch. & Dudheli & Ulmaceae & Tree \\
\hline 59 & Hydrilla verticillata (L. f.) Royle & $\begin{array}{l}\text { Hydrilla, Indian star } \\
\text { vine }\end{array}$ & Hydrocharitaceae & Herb \\
\hline 60 & Impatiens balsamina L. & Gulmehdi & Balsaminaceae & Herb \\
\hline 61 & Indigofera linifolia Retz. & Jinkigali & Fabaceae & Herb \\
\hline 62 & Indigofera tinctoria L. & Gali & Fabaceae & Herb \\
\hline 63 & Ipomoea aquatica Forssk. & Nali ni bhaji & Convolvulaceae & $\begin{array}{l}\text { Climbe } \\
\mathrm{r}\end{array}$ \\
\hline 65 & Ipomoea cairica (L) Sw. & Narvel & Convolvulaceae & $\begin{array}{l}\text { Climbe } \\
\mathrm{r}\end{array}$ \\
\hline 67 & Jatropha gossypifolia L. & Bellyache bush & Euphorbiaceae & Shrub \\
\hline 68 & $\begin{array}{l}\text { Lantana camara L. var. aculeata } \\
\text { (L.) Mold. }\end{array}$ & Gandhari & Verbenaceae & Shrub \\
\hline 69 & Lepidagathis trinervis Nees & Harancharo & Acanthaceae & Herb \\
\hline 70 & $\begin{array}{l}\text { Leucaena leucocephala (Lam.) de } \\
\text { Wit }\end{array}$ & White leadtree & Mimosaceae & Herb \\
\hline 71 & Luffa acutangula (L) Roxb & Turiya & Cucurbitaceae & $\begin{array}{l}\text { Climbe } \\
\mathrm{r}\end{array}$ \\
\hline 72 & Marselia spp. & Water Clover & Marseliaceae & $\begin{array}{l}\text { Pterido } \\
\text { phyte }\end{array}$ \\
\hline 73 & Martynia annua L. & Tiger's Claw & Martyniaceae & Herb \\
\hline
\end{tabular}


Juhi Gajjar et al Int J Sci Res Sci \& Technol. May-June-2021, 8 (3) : 259-266

\begin{tabular}{|c|c|c|c|c|}
\hline 74 & Melia azedarach L. & $\begin{array}{l}\text { Bakani Limbdo, Persian } \\
\text { Lilac }\end{array}$ & Meliaceae & Tree \\
\hline 75 & Najas marina L. & Spiny Water Nymph & Najadaceae & Herb \\
\hline 76 & Nelumbo nucifera Gaertn. & Indian Lotus & Nelumbaceae & Herb \\
\hline 77 & Nymphaea pubescens Willd. & Water Lily & Nymphaeaceae & Herb \\
\hline 78 & Nymphoides indica (L.) Kuntze & Water Snowflake & Menyanthaceae & Herb \\
\hline 79 & Ocimum gratissimum L & Mala tulsi & Lamiaceae & Herb \\
\hline 80 & Ocimum gratissimum L & Mala tulsi & Lamiaceae & Shrub \\
\hline 81 & Oldenlandia corymbosa L. & $\begin{array}{l}\begin{array}{l}\text { Diamond } \\
\text { Flower, } \\
\text { corymbose } \\
\text { hedyotis, }\end{array} \\
\text { flat-top mille grains }\end{array}$ & Rubiaceae & Herb \\
\hline 82 & $\begin{array}{l}\text { Operculina turpethum (L.) Silva } \\
\text { Manso }\end{array}$ & $\begin{array}{ll}\text { Indian } & \text { Jalap/ } \\
\text { Transparent } & \text { Wood } \\
\text { Rose } & \end{array}$ & Convolvulaceae & $\begin{array}{l}\text { Climbe } \\
\mathrm{r}\end{array}$ \\
\hline 83 & Opuntia elatior Mill & Prickly Pear & Cactaceae & Shrub \\
\hline 84 & Ottelia alismoides (L.) Persoon & Duck lettuce & Hydrocharitaceae & Herb \\
\hline 85 & Oxystelma esculentum (L. f.) Sm. & Jaldudhi & Asclepiadaceae & $\begin{array}{l}\text { Climbe } \\
\mathrm{r}\end{array}$ \\
\hline 86 & Panicum spp & Panicgrass & Poaceae & Grass \\
\hline 87 & Parthenium hysterophorus L. & Congress grass & Asteraceae & Herb \\
\hline 88 & $\begin{array}{lll}\text { Pergularia } & \text { daemia } & \text { (Forssk) } \\
\text { Chiov } & & \\
\end{array}$ & Chamar dudheli & Asclepiadaceae & $\begin{array}{l}\text { Climbe } \\
\text { r }\end{array}$ \\
\hline 89 & $\begin{array}{l}\text { Peristrophe paniculata (Forssk) } \\
\text { Brummitt }\end{array}$ & Kali aghedi & Acanthaceae & Shrub \\
\hline 90 & $\begin{array}{l}\text { Phyllanthus erectus (Medik.) } \\
\text { M.R.Almeida }\end{array}$ & $\begin{array}{l}\text { Stone-breaker, Seed } \\
\text { under leaf }\end{array}$ & Euphorbiaceae & Herb \\
\hline 91 & Phyllanthus reticulatus Poir & Kamboi & Euphorbiaceae & Shrub \\
\hline 92 & Physalis sp. & Berry & Solanaceae & Shrub \\
\hline 93 & Pluchea tomentosa DC. & $\begin{array}{l}\text { Wolly Champhor- } \\
\text { Weed }\end{array}$ & Asteraceae & Herb \\
\hline 94 & Plumbago auriculata Lam. & Nila chitrak & Plumbaginaceae & Shrub \\
\hline 95 & Pongamia pinnata (L.) Pierre & Karanj, Kanajhi & Fabaceae & Tree \\
\hline 96 & Potamogeton nodosus Poir. & Longleaf Pond Weed & Potamogetonaceae & Herb \\
\hline 97 & Potamogeton perfoliatus L. & Perfoliate Pond weed & Potamogetonaceae & Herb \\
\hline 98 & Prosopis julifera (Sw.) DC. & Gandobaval & Mimosaceae & Tree \\
\hline 99 & $\begin{array}{l}\text { Pupalia lappacea (L.) Juss. var. } \\
\text { lappacea }\end{array}$ & Nagadamani & Amaranthaceae & Herb \\
\hline 100 & Rivea hypocrateriformis Choisy & Common Night Glory & Convolvulaceae & $\begin{array}{l}\text { Climbe } \\
\text { r }\end{array}$ \\
\hline 101 & Salvadora persica L. & Piludi & Salvadoraceae & Shrub \\
\hline 102 & Senna alata (L.) Roxb. & Candle bush & Caesalpinaceae & Shrub \\
\hline
\end{tabular}


Juhi Gajjar et al Int J Sci Res Sci \& Technol. May-June-2021, 8 (3) : 259-266

\begin{tabular}{|c|c|c|c|c|}
\hline 103 & $\begin{array}{l}\text { Senna siamea (Lam.) H. S. Irwin } \\
\text { \& Barneby }\end{array}$ & Kassod & Caesalpinaceae & Tree \\
\hline 104 & Senna auriculata (L.) Roxb. & Awala, Tarwar & Caesalpinaceae & Shrub \\
\hline 105 & Senna occidentalis (L.) Link & Coffeeweed & Caesalpinaceae & Shrub \\
\hline 106 & Senna tora (L.) Roxb. & Stinking cassia & Caesalpinaceae & Herb \\
\hline 107 & Sesamum indicum L. & Safedtil & Pedaliaceae & Herb \\
\hline 108 & Setaria italica (L) P Beauv & Chano & Poaceae & Herb \\
\hline 109 & Sida acuta Burm $\mathrm{f}$ & Bala & Malvaceae & Herb \\
\hline 110 & Sida orientalis Cav & Bala & Malvaceae & Herb \\
\hline 111 & Sida rhombifolia L. & Heart-leaf, Sahadeva & Malvaceae & Herb \\
\hline 112 & $\begin{array}{l}\text { Sida cordata (Burm.f.) } \\
\text { Borss.Waalk. }\end{array}$ & Bhuyinii, Bhoybala & Malvaceae & Herb \\
\hline 113 & Solanum indicum L & Jangli ringni & Solanaceae & Shrub \\
\hline 114 & Solanum nigrum L & Piludi & Solanaceae & Shrub \\
\hline 115 & Solanum diphyllum L. & Twoleaf nightshade & Solanaceae & Shrub \\
\hline 116 & Spermacoce articularis L.F. & False buttenweed & Rubiacaeae & Herb \\
\hline 117 & Stuckenia pectinata (L.) Börner & Fennel Pondweed & Potamogetonaceae & Herb \\
\hline 118 & Teramnus labialis (L f) Spreng & Ran-udid & Fabaceae & $\begin{array}{l}\text { Climbe } \\
\mathrm{r}\end{array}$ \\
\hline 119 & Teramnus mollis Benth. & $\begin{array}{l}\text { Blue wiss, Horse wine, } \\
\text { Rabbit wine }\end{array}$ & Fabaceae & $\begin{array}{l}\text { Climbe } \\
\mathrm{r}\end{array}$ \\
\hline 120 & Trianthema portulacastrum L & & Aizoaceae & Herb \\
\hline 121 & Tribulus terrestris $\mathrm{L}$ & Bethu Gokhru & Zygophyllaceae & Herb \\
\hline 122 & $\begin{array}{l}\text { Trichodesma indicum (L.) Lehm. } \\
\text { var. indicum }\end{array}$ & Adhapushpi & Boraginaceae & Herb \\
\hline 123 & Typha latifolia & Common cattail & Typhaceae & Herb \\
\hline 124 & Xanthium strumarium L. & Gokhru & Asteraceae & Herb \\
\hline 125 & Ziziphus rugosa Lam & Suran & Rhamnaceae & Shrub \\
\hline
\end{tabular}

Study revels about 125 species of angiosperms and one species of Pteridophytes. These 125 species belong to 97 Genera and 46 Family. Study represents 14 tree 18 shrub, 5 climbers 68 herb, 4 sedges, and 5 grass species.

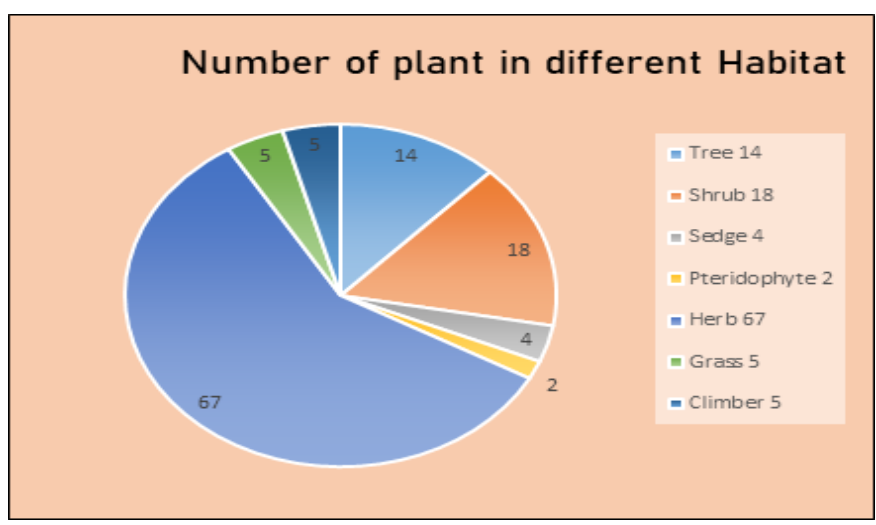

Graph: 1: Number of plants in different Habitat 


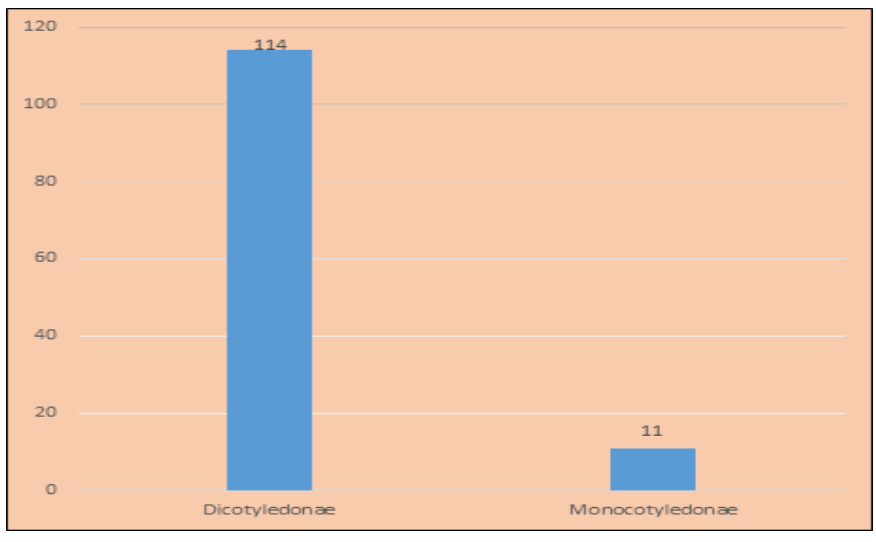

Graph: 2: Number of species in Class Dicotyledonous and Class Monocotyledon

\section{IV.CONCLUSION}

Vegetation of Kanewal wetland shows the diversity of different plant species. Study revels about 125 species of angiosperms and one species of Pteridophytes. These 125 species belong to 97 Genera and 46 Family. Study represents 14 trees 18 shrubs, 5 climbers 68 herbs, 4 sedges, and 5 grasses species. Mostly there are vegetation of Typha is observed. Eichhornia crassipes is very fast growing there. It may cause eutrophication to wetland. Which is harmful for aquatic plant species and other aquatic animals. During the summer water level decreases at that time increasing in algal blooms, which cover the upper layer of the water. Many medicinally important species were found there. It is necessary to protect the land from excessive cutting and grazing of animals.

\section{ACKNOWLEDGEMENT}

I would like to say special thanks to Dr.Rupesh Maurya for identification of plants.

\section{REFERENCES}

[1]. Allen-Diaz, B., R. D. Jackson, K. W. Tate, and L. G. Oates. 2004. California Agriculture 58(3): 144148.

[2]. Ghermandi, A., van den Bergh, J.C.J.M., Brander, L.M., Nunes, P.A.L.D., (2008). The Economic Value of Wetland Conservation and Creation: A Meta-Analysis. [Working Paper 79]. Fondazione Eni Enrico Mattei, Milan, Italy.

[3]. Zedler, J.B., Kercher, S., (2005). Wetland resources: status, trends, ecosystem services, and restorability. Annu. Rev. Environ.Resour. 30 (1), 39-74.

\section{Cite this article as :}

Juhi Gajjar, Dr. Hitesh Solanki, "Vegetation Analysis of Kanewal Wetland, Gujarat", International Journal of Scientific Research in Science and Technology (IJSRST), Online ISSN : 2395-602X, Print ISSN : 23956011, Volume 8 Issue 3, pp. 259-266, May-June 2021. Available at

doi : https://doi.org/10.32628/IJSRST218341

Journal URL : https://ijsrst.com/IJSRST218341 